\title{
HOMOTOPY PERTURBATION SHEHU TRANSFORM METHOD FOR SOLVING FRACTIONAL MODELS ARISING IN APPLIED SCIENCES
}

\author{
Shehu Maitama, Weidong Zhao \\ School of Mathematics, Shandong University, Jinan \\ Shandong, China \\ smusman12@sci.just.edu.jo,wdzhao@sdu.edu.cn
}

Received: 10 November 2020; Accepted: 17 March 2021

\begin{abstract}
Using the recently proposed homotopy perturbation Shehu transform method (HPSTM), we successfully construct reliable solutions of some important fractional models arising in applied physical sciences. The nonlinear terms are decomposed using He's polynomials, and the fractional derivative is calculated in the Caputo sense. Using the analytical method, we obtained the exact solution of the fractional diffusion equation, fractional wave equation and the nonlinear fractional gas dynamic equation.
\end{abstract}

MSC 2010: 34K50, 34A12, 34A30, 45A05, 44A05, 44A20

Keywords: homotopy perturbation technique, Shehu transform, fractional models, numeric and symbolic computations

\section{Introduction}

In recent years, the concept of calculus has been widely studied due to their applicability in modeling various important applications in applied physical science. The concept of non-integer-order models was used to model applications in signal processing, fluid mechanics, acoustics, electromagnetism, analytical chemistry, biology, and many other useful areas of engineering [1-5].

Recently, many fractional models have been solved using analytical and numerical techniques. To mention a few, we have the homotopy perturbation method (HPM) [6], the Adomian decomposition method (ADM) [7], the Laplace decomposition method (LDM) [8], the homotopy perturbation transform method (HPTM) [9], and so on. Besides using the Laplace-type integral transform [10,11], some new efficient iterative techniques with the Caputo fractional derivative [12] and Atangana-Baleanu fractional derivative [13] are developed, for example, see [14-25]. Those iterative algorithms are successfully applied to many applications in applied physical science.

The aim of this work is to further apply the homotopy perturbation Shehu transform method (HPSTM) to some useful fractional models arising in real-life problems. The HPSTM is applied directly to fractional models without any discretiza- 
tion, linearization, or variable transformations. The HPSTM is an iterative technique which converges to solutions in closed form or approximate solutions. The nonlinear terms are decomposed successfully via He's polynomials, and the fractional derivatives are computed in the Caputo sense. Applications of three fractional models are demonstrated, and the analytical and numerical simulations of the three fractional models are provided to buttress the efficiency, simplicity and the high accuracy of the HPSTM. The remaining sections of the paper are organized as follows. In section 2, some preliminaries used in this paper are given. The algorithm of HPSTM and convergence are presented in section 3. Applications of the HPSTM are given in section 4 . The conclusion is given in section 5 .

\section{Preliminaries}

Here, we present the definition and properties of the STM which generalize the well-known Laplace transform and the Sumudu integral transform.

DEFINITION $1[10,11]$ The Shehu transformation of the function $q(t)$ of exponential order is defined over the set of functions,

$\mathscr{A}=\left\{q(t): \exists \mathscr{C}, \omega_{1}, \omega_{2}>0,|q(t)|<\mathscr{C} \exp \left(\frac{|t|}{\omega_{k}}\right)\right.$, if $\left.t \in(-1)^{k} \times[0, \infty)\right\}$,

by the following integral

$$
\mathbb{S}[q(t)]=\mathscr{Q}(\zeta, \xi)=\int_{0}^{\infty} \exp \left(\frac{-\zeta t}{\xi}\right) q(t) d t, \zeta>0, \xi>0 .
$$

And the inverse STM is defined as

$$
\mathbb{S}^{-1}[\mathscr{Q}(\zeta, \xi)]=q(t), \text { forall } t \geq 0 .
$$

Equivalently

$$
q(t)=\mathbb{S}^{-1}[\mathscr{Q}(\zeta, \xi)]=\frac{1}{2 \pi i} \int_{\vartheta-i \infty}^{\vartheta+i \infty} \frac{1}{\xi} \exp \left(\frac{\zeta t}{\xi}\right) \mathscr{Q}(\zeta, \xi) d \zeta,
$$

where $\zeta$ and $\xi$ are STM variables, and $\vartheta$ is a real constant and the integral in Eq. (3) is taken along $\zeta=\vartheta$ in the complex plane $\zeta=x+i y$.

PROPERTY 1 [10,11] The STM of nth-order derivatives with respect to $t$ is defined as

$$
\mathbb{S}\left[q^{(n)}(t)\right]=\frac{\zeta^{n}}{\xi^{n}} \mathbb{S}[q(t)]-\sum_{m=0}^{m-1}\left(\frac{\zeta}{\xi}\right)^{m-(j+1)} q^{(j)}(0)
$$


PROPERTY 2 [10-12] The STM of the Caputo fractional derivative is defined as

$$
\mathbb{S}\left[{ }^{C} D_{t}^{\alpha} q(t)\right]=\left(\frac{\zeta}{\xi}\right)^{\alpha} \mathbb{S}[q(t)]-\sum_{j=0}^{j-1}\left(\frac{\zeta}{\xi}\right)^{\alpha-j-1} q^{(j)}(0+), j-1<\alpha \leq j .
$$

DEFINITION 2 [23] The Caputo fractional derivative of the function $q(t)$ of order $\alpha$ is defined as

$$
C_{D_{t}^{\alpha}}^{\alpha} q(t)=\left\{\begin{array}{l}
\frac{1}{\Gamma(m-\alpha)} \int_{0}^{t}(t-\eta)^{m-\alpha-1} q^{(m)}(\eta) d \eta \\
I^{m-\beta} D^{m} q(t), \text { where } m=[\alpha]+1
\end{array}\right.
$$

Definition 3 [23] The Mittag-Leffler function for one parameter is defined by the series expansion

$$
E_{\mu}(\lambda)=\sum_{k=0}^{\infty} \frac{\lambda^{k}}{\Gamma(\mu k+1)}, \mathbb{R}(\mu)>0, \mu, \lambda \in \mathbb{C}
$$

\section{Algorithm of the HPSTM}

The algorithm of the HPSTM on a standard nonlinear fractional model with initial condition (IC) is illustrated in the following section.

$$
D_{\psi}^{\alpha} v(\mu, \psi)+\mathscr{M}(v(\mu, \psi))+\mathscr{F}(v(\mu, \psi))=\mathscr{G}(\mu, \psi),
$$

with the initial condition

$$
v(\mu, 0)=f(\mu)
$$

where $\mathscr{F}(v(\mu, \psi))$ stand for the nonlinear operator, $D_{\psi}^{\alpha}=\frac{\partial^{\alpha}}{\partial \psi^{\alpha}}$ denote the Caputo fractional operator, $\mathscr{M}(v(\mu, \psi))$ is the linear differential operator, and $\mathscr{G}(\mu, \psi)$ is a source term.

Computing STM on Eq. (8) gives

$V(\mu, \zeta, \xi)=\frac{\xi}{\zeta} f(\mu)+\left(\frac{\xi}{\zeta}\right)^{\alpha} \mathbb{S}[\mathscr{G}(\mu, \psi)]-\left(\frac{\xi}{\zeta}\right)^{\alpha} \mathbb{S}[\mathscr{M}(v(\mu, \psi))+\mathscr{F}(v(\mu, \psi))]$.

Inverting Eq. (10), we get

$$
v(\mu, \psi)=\mathscr{G}(\mu, \psi)-\mathbb{S}^{-1}\left[\left(\frac{\xi}{\zeta}\right)^{\alpha} \mathbb{S}[\mathscr{M}(v(\mu, \psi))+\mathscr{F}(v(\mu, \psi))]\right],
$$


where $\mathscr{G}(\mu, \psi)=\mathbb{S}^{-1}\left[\frac{\xi}{\zeta} f(\mu)+\left(\frac{\xi}{\zeta}\right)^{\alpha} \mathbb{S}[\mathscr{G}(\mu, \psi)]\right.$.

Then applying HPM, we get

$$
v(\mu, \psi)=\sum_{n=0}^{\infty} \Theta^{n} v_{n}(\mu, \psi) .
$$

We decomposed the nonlinear term $\mathscr{F}(v(\mu, \psi))$ with

$$
\mathscr{F}(v(\mu, \psi))=\sum_{n=0}^{\infty} \Theta^{n} \mathscr{H}_{n}(v),
$$

where $\mathscr{H}_{n}(v)$ is the He's polynomials which is defined as

$$
\mathscr{H}_{n}\left(v_{1}, v_{2}, \cdots, v_{n}\right)=\frac{1}{n !} \frac{\partial^{n}}{\partial \Theta^{n}}\left[\mathscr{F}\left(\sum_{j=0}^{n} \Theta^{j} v_{j}\right)\right]_{\Theta=0}, n=0,1,2, \cdots
$$

After substitution, Eq. (11) gives

$$
\begin{aligned}
\sum_{n=0}^{\infty} \Theta^{n} v_{n}(\mu, \psi)= & \mathscr{G}(\mu, \psi)-\Theta\left(\mathbb { S } ^ { - 1 } \left[( \frac { \xi } { \zeta } ) ^ { \alpha } \mathbb { S } \left[\sum_{n=0}^{\infty} \Theta^{n} \mathscr{M}\left(v_{n}(\mu, \psi)\right)\right.\right.\right. \\
& \left.\left.\left.+\sum_{n=0}^{\infty} \Theta^{n} \mathscr{H}_{n}(v)\right]\right]\right)
\end{aligned}
$$

Using the coefficients of the same powers of $\Theta$ in Eq. (14), we get

$$
\begin{aligned}
\Theta^{0}: v_{0}(\mu, \psi) & =\mathscr{G}(\mu, \psi) \\
\Theta^{1}: v_{1}(\mu, \psi) & =-\mathbb{S}^{-1}\left[\left(\frac{\xi}{\zeta}\right)^{\alpha} \mathbb{S}\left[\mathscr{M}\left(v_{0}(\mu, \psi)\right)+\mathscr{H}_{0}(v)\right]\right], \\
\Theta^{2}: v_{2}(\mu, \psi) & =-\mathbb{S}^{-1}\left[\left(\frac{\xi}{\zeta}\right)^{\alpha} \mathbb{S}\left[\mathscr{M}\left(v_{1}(\mu, \psi)\right)+\mathscr{H}_{1}(v)\right]\right], \\
\vdots & \\
\Theta^{n}: v_{n}(\mu, \psi) & =-\mathbb{S}^{-1}\left[\left(\frac{\xi}{\zeta}\right)^{\alpha} \mathbb{S}\left[\mathscr{M}\left(v_{n-1}(\mu, \psi)\right)+\mathscr{H}_{n-1}(v)\right]\right], n>0, n \in \mathscr{N} .
\end{aligned}
$$

Finally, as $\Theta \rightarrow 1$, we get

$$
v(\mu, \psi)=\lim _{N \rightarrow \infty} \sum_{n=0}^{N} v_{n}(\mu, \psi) .
$$

In the following theorem, we prove the convergence analysis and the error analysis of the HPSTM.

THeORem 1 Convergence analysis. Let $\mathscr{X}$ be a Banach space and let $\zeta_{m}(\mu, \psi)$ 
and $\left.\zeta_{(} \mu, \psi\right)$ be in $\mathscr{X}$. Suppose $\Theta \in(0,1)$, then the series solution $\left\{\zeta_{m}(\mu, \psi)\right\}_{m=0}^{\infty}$ which is defined from $\sum_{m=0}^{\infty} \zeta_{m}(\mu, \psi)$ converges to the solution of Eq. (8) whenever $\zeta_{m}(\mu, \psi) \leq \Theta \zeta_{m-1}(\mu, \psi) \forall m>\mathbb{N}$, that is for any given $\varepsilon>0$ there exists a positive number $\mathbb{N}$ such that $\left\|\zeta_{m+n}(\mu, \psi)\right\| \leq \varepsilon \forall m, n>\mathbb{N}$.

Besides, the absolute error is

$$
\left\|\zeta(\mu, \psi)-\sum_{n=0}^{m} \zeta_{n}(\mu, \psi)\right\| \leq \frac{\Theta^{m+1}}{1-\Theta}\left\|\zeta_{0}(\mu, \psi)\right\| .
$$

PROOF Let us first define a sequence of partial sum $\left\{\mathfrak{I}_{m}(\mu, \psi)\right\}_{m=0}^{\infty}$ as

$$
\begin{aligned}
\mathfrak{I}_{0}(\mu, \psi) & =\zeta_{0}(\mu, \psi), \\
\mathfrak{I}_{1}(\mu, \psi) & =\zeta_{0}(\mu, \psi)+\zeta_{1}(\mu, \psi), \\
\mathfrak{I}_{2}(\mu, \psi) & =\zeta_{0}(\mu, \psi)+\zeta_{1}(\mu, \psi)+\zeta_{2}(\mu, \psi), \\
\vdots & \\
\mathfrak{I}_{m}(\mu, \psi) & =\zeta_{0}(\mu, \psi)+\zeta_{1}(\mu, \psi)+\zeta_{2}(\mu, \psi)+\zeta_{3}(\mu, \psi)+\ldots+\zeta_{m}(\mu, \psi) .
\end{aligned}
$$

We only need to show that $\mathfrak{I}_{m}(\mu, \psi)$ is a Cauchy sequence in $\mathscr{X}$. To prove the claim, since $\Theta \in(0,1)$, the following inequality holds

$$
\begin{aligned}
& \left\|\mathfrak{I}_{m+1}(\mu, \psi)-\mathfrak{I}_{m}(\mu, \psi)\right\|=\left\|\zeta_{m+1}(\mu, \psi)\right\| \leq \Theta\left\|\zeta_{m}(\mu, \psi)\right\| \leq \Theta^{2}\left\|\zeta_{m-1}(\mu, \psi)\right\| \\
& \leq \Theta^{3}\left\|\zeta_{m-2}(\mu, \psi)\right\| \leq \Theta^{4}\left\|\zeta_{m-3}(\mu, \psi)\right\| \leq \cdots \leq \Theta^{m+1}\left\|\zeta_{0}(\mu, \psi)\right\|
\end{aligned}
$$

Then for any $m, n \in \mathbb{N}, n>m$, we obtain

$$
\begin{aligned}
& \left\|\mathfrak{I}_{m}(\mu, \psi)-\mathfrak{I}_{n}(\mu, \psi)\right\|=\left\|\zeta_{m+n}(\mu, \psi)\right\|=\|\left(\mathfrak{I}_{m}(\mu, \psi)-\mathfrak{I}_{m-1}(\mu, \psi)\right) \\
& +\left(\mathfrak{I}_{m-1}(\mu, \psi)-\mathfrak{I}_{m-2}(\mu, \psi)\right)+\left(\mathfrak{I}_{m-2}(\mu, \psi)-\mathfrak{I}_{m-3}(\mu, \psi)\right) \\
& +\cdots+\left(\mathfrak{I}_{n+1}(\mu, \psi)-\mathfrak{I}_{n}(\mu, \psi)\right)\|\leq\| \mathfrak{I}_{m}(\mu, \psi)-\mathfrak{I}_{m-1}(\mu, \psi) \| \\
& +\left\|\mathfrak{I}_{m-1}(\mu, \psi)-\mathfrak{I}_{m-2}(\mu, \psi)\right\|+\left\|\mathfrak{I}_{m-2}(\mu, \psi)-\mathfrak{I}_{m-3}(\mu, \psi)\right\| \\
& +\cdots+\left\|\mathfrak{I}_{n+1}(\mu, \psi)-\mathfrak{I}_{n}(\mu, \psi)\right\| \leq \Theta^{m}\left\|\zeta_{0}(\mu, \psi)\right\|+\Theta^{m-1}\left\|\zeta_{0}(\mu, \psi)\right\| \\
& +\Theta^{m-2}\left\|\zeta_{0}(\mu, \psi)\right\|+\Theta^{m-3}\left\|\zeta_{0}(\mu, \psi)\right\|+\cdots+\Theta^{m+1}\left\|\zeta_{0}(\mu, \psi)\right\| \\
= & \left\|\zeta_{0}(\mu, \psi)\right\| \frac{1-\Theta^{m-n}}{1-\Theta} \Theta^{n+1} .
\end{aligned}
$$

Since $\Theta \in(0,1), 1>1-\Theta^{m-n}$ and $\zeta_{0}(\mu, \psi)$ is bounded, we obtain

$$
\left\|\zeta_{m+n}(\mu, \psi)\right\| \leq \varepsilon \forall n, m>\mathbb{N}, \text { or } \lim _{m, n \rightarrow \infty}\left\|\zeta_{m+n}(\mu, \psi)\right\|=0
$$


Thus, the sequence $\left\{\mathfrak{I}_{m}(\mu, \psi)\right\}_{m=0}^{\infty}$ is a Cauchy sequence in $\mathscr{X}$. The error estimate follows directly from Theorem 1 and inequality (17). This completes the proof.

\section{Demonstrated examples}

Application of the HPSTM are illustrated in this section to verify its applicability and accuracy.

EXAMPLE 1 Consider the wave-like one-dimensional fractional model

$$
D_{t}^{\alpha} v-v_{x x}-v=0,
$$

with the initial condition

$$
v(x, 0)=\cos (\pi x), \quad \alpha \in(0,1) .
$$

Applying STM on Eq. (20) yields

$$
V(x, \zeta, \xi)=\frac{\xi}{\zeta} \cos (\pi x)+\left(\frac{\xi}{\zeta}\right)^{\alpha} \mathbb{S}\left[v_{x x}+v\right] .
$$

Inverting Eq. (22), we obtain

$$
v(x, t)=\cos (\pi x)+\mathbb{S}^{-1}\left[\left(\frac{\xi}{\zeta}\right)^{\alpha} \mathbb{S}\left[v_{x x}+v\right]\right] .
$$

Based on the HPM algorithm, we get

$$
v(x, t)=\sum_{n=0}^{\infty} \Theta^{n} v_{n}(x, t) .
$$

Thus Eq. (23) becomes

$$
\sum_{n=0}^{\infty} \Theta^{n} v_{n}(x, t)=\cos (\pi x)+\Theta\left(\mathbb{S}^{-1}\left[\left(\frac{\xi}{\zeta}\right)^{\alpha} \mathbb{S}\left[\sum_{n=0}^{\infty} \Theta^{n} v_{n x x}+\sum_{n=0}^{\infty} \Theta^{n} v_{n}\right]\right]\right) .
$$

From the coefficients of the same powers of $\Theta$ in Eq. (25), we get

$$
\begin{aligned}
& \Theta^{0}: v_{0}(x, t)=\cos (\pi x) \\
& \Theta^{1}: v_{1}(x, t)=\mathbb{S}^{-1}\left[\left(\frac{\xi}{\zeta}\right)^{\alpha} \mathbb{S}\left[v_{0 x x}+v_{0}\right]\right]=\left(1-\pi^{2}\right) \cos (\pi x) \frac{t^{\alpha}}{\Gamma(\alpha+1)}, \\
& \Theta^{2}: v_{2}(x, t)=\mathbb{S}^{-1}\left[\left(\frac{\xi}{\zeta}\right)^{\alpha} \mathbb{S}\left[v_{1 x x}+v_{1}\right]\right]=\left(1-\pi^{2}\right)^{2} \cos (\pi x) \frac{t^{2 \alpha}}{\Gamma(2 \alpha+1)}
\end{aligned}
$$


Thus, as $\Theta \rightarrow 1$, we get

$$
\begin{aligned}
v(x, t) & =v_{0}(x, t)+v_{1}(x, t)+v_{2}(x, t)+v_{3}(x, t)+\cdots \\
& =\cos (\pi x)\left(1+\frac{\left(1-\pi^{2}\right) t^{\alpha}}{\Gamma(\alpha+1)}+\frac{\left(\left(1-\pi^{2}\right) t^{\alpha}\right)^{2}}{\Gamma(2 \alpha+1)}+\frac{\left(\left(1-\pi^{2}\right) t^{\alpha}\right)^{3}}{\Gamma(3 \alpha+1)}+\cdots\right) \\
& =\cos (\pi x) \sum_{m=0}^{\infty} \frac{\left(\left(1-\pi^{2}\right) t^{\alpha}\right)^{m}}{\Gamma(m \alpha+1)}=E_{\alpha}\left(\left(1-\pi^{2}\right) t^{\alpha}\right) \cos (\pi x),
\end{aligned}
$$

where $E_{\alpha}($.$) is the Mittag-Leffler function. The special case of \alpha=1$ will give the following result

$$
v(x, t)=\exp \left(\left(1-\pi^{2}\right) t\right) \cos (\pi x),
$$

which is the solution in closed form [25].

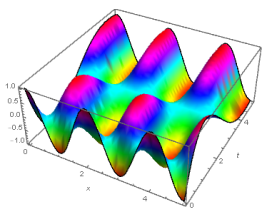

(a)

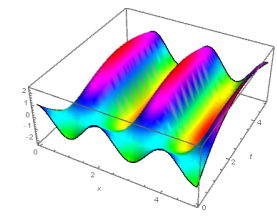

(b)

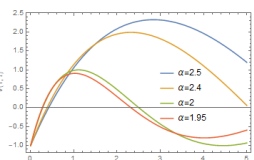

(c)

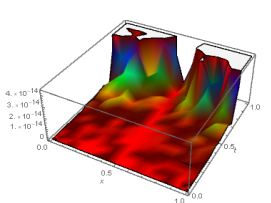

(d)

Fig. 1. (a) Exact solution of Eq. (20) for $\alpha=2$ preserves a clear wave and diffusion behavior. The behavior is maintained in [25]. The approximate solution behavior of Eq. (20) when $\alpha=2.5$ is plotted in Fig. 1(b), and the increase in the value of $\alpha$ results in increase in diffusion and wave behavior. The (c) exact and approximate solutions of Eq. (20) for different time intervals and different

$\alpha^{\prime} s$ are presented in Fig. 1(c). (d) Error analysis of $10^{\text {th }}$-order approximations of Example 1

EXAMPLE 2 Consider the wave-like three-dimensional fractional model

$$
D_{t}^{\alpha} v-\frac{1}{36}\left(x^{2} v_{x x}+y^{2} v_{y y}+z^{2} v_{z z}\right)=(x y z)^{4}, 0<x, y, z<1,0<\alpha \leq 1,
$$

with the initial condition

$$
v(x, y, z, 0)=0 .
$$

Applying STM on Eq. (28), we get

$$
V(x, y, z, \zeta, \xi)=\left(\frac{\xi}{\zeta}\right)^{\alpha} \mathbb{S}\left[(x y z)^{4}+\frac{1}{36}\left(x^{2} v_{x x}+y^{2} v_{y y}+z^{2} v_{z z}\right)\right]
$$

Inverting Eq. (30), we have

$$
v(x, y, z, t)=\mathbb{S}^{-1}\left[\left(\frac{\xi}{\zeta}\right)^{\alpha} \mathbb{S}\left[(x y z)^{4}+\frac{1}{36}\left(x^{2} v_{x x}+y^{2} v_{y y}+z^{2} v_{z z}\right)\right]\right] .
$$


According to HPM, we have

$$
v(x, y, z, t)=\sum_{n=0}^{\infty} \Theta^{n} v_{n}(x, y, z, t)
$$

Thus, Eq. (31) becomes

$$
\begin{aligned}
\sum_{n=0}^{\infty} \Theta^{n} v_{n}(x, y, z, t)= & \Theta\left(\mathbb { S } ^ { - 1 } \left[( \frac { \xi } { \zeta } ) ^ { \alpha } \mathbb { S } \left[(x y z)^{4}+\frac{1}{36}\left(x^{2} \sum_{n=0}^{\infty} \Theta^{n} v_{n x x}\right.\right.\right.\right. \\
& \left.\left.\left.\left.+y^{2} \sum_{n=0}^{\infty} \Theta^{n} v_{n y y}+z^{2} \sum_{n=0}^{\infty} \Theta^{n} v_{n z z}\right)\right]\right]\right) .
\end{aligned}
$$

Using the coefficients of the same powers of $\Theta$ in Eq. (33), we get

$$
\begin{aligned}
\Theta^{0}: v_{0}(x, y, z, t) & =0 \\
\Theta^{1}: v_{1}(x, y, z, t) & =\mathbb{S}^{-1}\left[\left(\frac{\xi}{\zeta}\right)^{\alpha} \mathbb{S}\left[(x y z)^{4}+\frac{1}{36}\left(x^{2} v_{0 x x}+y^{2} v_{0 y y}+z^{2} v_{0 z z}\right)\right]\right] \\
& =(x y z)^{4} \frac{t^{\alpha}}{\Gamma(\alpha+1)}, \\
\Theta^{2}: v_{2}(x, y, z, t) & =\mathbb{S}^{-1}\left[\left(\frac{\xi}{\zeta}\right)^{\alpha} \mathbb{S}\left[(x y z)^{4}+\frac{1}{36}\left(x^{2} v_{1 x x}+y^{2} v_{1 y y}+z^{2} v_{1 z z}\right)\right]\right] \\
& =(x y z)^{4} \frac{t^{2 \alpha}}{\Gamma(2 \alpha+1)}
\end{aligned}
$$

Thus as $\Theta \rightarrow 1$, we have

$$
\begin{aligned}
v(x, y, z, t) & =v_{0}(x, y, z, t)+v_{1}(x, y, z, t)+v_{2}(x, y, z, t)+v_{3}(x, y, z, t)+\cdots \\
& =(x y z)^{4}\left(\frac{t^{\alpha}}{\Gamma(\alpha+1)}+\frac{t^{2 \alpha}}{\Gamma(2 \alpha+1)}+\frac{t^{3 \alpha}}{\Gamma(3 \alpha+1)}+\cdots\right) \\
& =(x y z)^{4} \sum_{m=1}^{\infty} \frac{\left(t^{\alpha}\right)^{m}}{\Gamma(m \alpha+1)}=(x y z)^{4}\left(E_{\alpha}\left(t^{\alpha}\right)-1\right),
\end{aligned}
$$

where $E_{\alpha}\left(t^{\alpha}\right)$ denotes the Mittag-Leffler function. The special case of $\alpha=1$ will give the following result

$$
v(x, y, z, t)=(x y z)^{4}(\exp (t)-1),
$$

which is the exact solution of the non-homogeneous linear fractional model in closed form. 


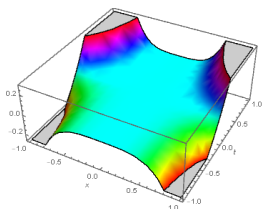

(a)

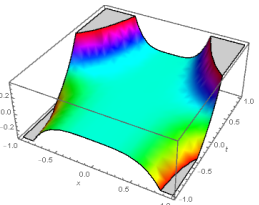

(b)

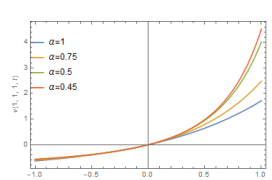

(c)

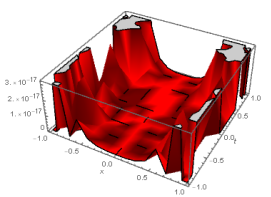

(d)

Fig. 2. (a) The surface solution of Eq. (28) for $\alpha=1$. The solution shows a clear wave and diffusion behavior. In Fig. 2(b), the approximate solution behavior of Eq. (28) when $\alpha=0.5$ is depicted and the diffusion and wave behavior decreases when the value of $\alpha$ is increased. In Fig. 2(c), the exact and approximate solutions of Eq. (28) for different time intervals and different $\alpha$ are depicted. The error analysis of $10^{\text {th }}$-order approximations of Example 2 is presented in Fig. 2(c)

EXAMPLE 3 Consider the following nonlinear fractional gas dynamic equation

$$
D_{t}^{\alpha} v+\frac{1}{2}\left(v^{2}\right)_{x}=v-v^{2}, 0<\alpha \leq 1, t>0,
$$

with the initial condition

$$
v(x, 0)=\exp (-x)
$$

Applying STM on Eq. (36), we have

$$
V(x, \zeta, \xi)=\frac{\xi}{\zeta} \exp (-x)+\left(\frac{\xi}{\zeta}\right)^{\alpha}\left[\mathbb{S}\left[v-\frac{1}{2}\left(v^{2}\right)_{x}-v^{2}\right]\right]
$$

Inverting Eq. (38), we get

$$
v(x, t)=\exp (-x)+\mathbb{S}^{-1}\left[\left(\frac{\xi}{\zeta}\right)^{\alpha}\left[\mathbb{S}\left[v-\frac{1}{2}\left(v^{2}\right)_{x}-v^{2}\right]\right]\right] .
$$

Now, based on the HPM, we get

$$
v(x, t)=\sum_{n=0}^{\infty} \Theta^{n} v_{n}(x, t) .
$$

Thus, Eq. (39) becomes

$$
\begin{aligned}
\sum_{n=0}^{\infty} \Theta^{n} v_{n}(x, t)= & \exp (-x)+\Theta\left(\mathbb { S } ^ { - 1 } \left[( \frac { \xi } { \zeta } ) ^ { \alpha } \left[\mathbb { S } \left[\sum_{n=0}^{\infty} \Theta^{n} v_{n}-\frac{1}{2} \sum_{n=0}^{\infty} \Theta^{n} \mathscr{H}_{n}^{\prime}(v)\right.\right.\right.\right. \\
& \left.\left.\left.\left.-\sum_{n=0}^{\infty} \Theta^{n} \mathscr{H}_{n}(v)\right]\right]\right]\right)
\end{aligned}
$$

where $\mathscr{H}_{n}(v)$ and $\mathscr{H}_{n}^{\prime}(v)$ are the He's polynomials denoting the nonlinear terms $v^{2}$ 
and $\left(v^{2}\right)_{x}$ respectively. Here are a few computed terms

$$
\begin{array}{rr}
\mathscr{H}_{0}^{\prime}(v)=\left(v_{0}^{2}\right)_{x}, & \mathscr{H}_{0}(v)=v_{0}^{2}, \\
\mathscr{H}_{1}^{\prime}(v)=\left(2 v_{0} v_{1}\right)_{x}, & \mathscr{H}_{1}(v)=2 v_{0} v_{1}, \\
\mathscr{H}_{2}^{\prime}(v)=\left(2 v_{0} v_{2}+v_{1}^{2}\right)_{x}, & \mathscr{H}_{2}(v)=2 v_{0} v_{2}+v_{1}^{2}, \\
\mathscr{H}_{3}^{\prime}(v)=\left(2 v_{0} v_{3}+2 v_{1} v_{2}\right)_{x}, & \mathscr{H}_{3}(v)=2 v_{0} v_{3}+2 v_{1} v_{2},
\end{array}
$$

Using the coefficients of the same powers of $\Theta$ in Eq. (41), we have

$$
\begin{aligned}
& \Theta^{0}: v_{0}(x, t)=\exp (-x) \\
& \Theta^{1}: v_{1}(x, t)=\mathbb{S}^{-1}\left[\left(\frac{\xi}{\zeta}\right)^{\alpha}\left[\mathbb{S}\left[v_{0}-\frac{1}{2} \mathscr{H}_{0}^{\prime}(v)-\mathscr{H}_{0}(v)\right]\right]\right]=\exp (-x) \frac{t^{\alpha}}{\Gamma(\alpha+1)} \\
& \Theta^{2}: v_{2}(x, t)=\mathbb{S}^{-1}\left[\left(\frac{\xi}{\zeta}\right)^{\alpha}\left[\mathbb{S}\left[v_{1}-\frac{1}{2} \mathscr{H}_{1}^{\prime}(v)-\mathscr{H}_{1}(v)\right]\right]\right]=\exp (-x) \frac{t^{2 \alpha}}{\Gamma(2 \alpha+1)}
\end{aligned}
$$

Thus, as $\Theta \rightarrow 1$, we get

$$
\begin{aligned}
v(x, t) & =v_{0}(x, t)+v_{1}(x, t)+v_{2}(x, t)+v_{3}(x, t)+\cdots \\
& =\exp (-x)\left(1+\frac{t^{\alpha}}{\Gamma(\alpha+1)}+\frac{t^{2 \alpha}}{\Gamma(2 \alpha+1)}+\frac{t^{3 \alpha}}{\Gamma(3 \alpha+1)}+\cdots\right) \\
& =\exp (-x) \sum_{m=0}^{\infty} \frac{\left(t^{\alpha}\right)^{m}}{\Gamma(m \alpha+1)}=\exp (-x) E_{\alpha}\left(t^{\alpha}\right),
\end{aligned}
$$

where $E_{\alpha}\left(t^{\alpha}\right)$ denotes the Mittag-Leffler function. The special case of $\alpha=1$ will give the following result in closed form

$$
v(x, t)=\exp (t-x) .
$$

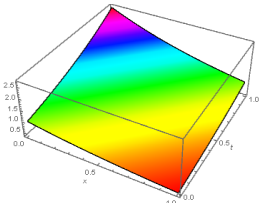

(a)

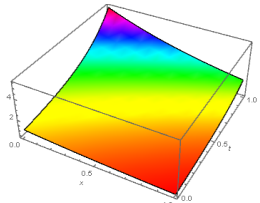

(b)

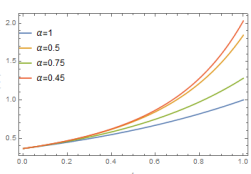

(c)

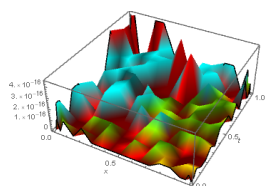

(d)

Fig. 3. The surface solution behavior of Eq. (36) when $\alpha=1$ is plotted in Fig. 3(a). In Fig. 3(b), the approximate solution behavior of Eq. (36) when $\alpha=0.5$ is presented. The exact and approximate solutions of Eq. (36) for different time intervals and a different $\alpha$ are depicted in Fig. 3(c).

The decrease in $\alpha$ exhibits slow diffusion behavior. The absolute error analysis of $10^{\text {th }}$-order approximations of Example 3 is presented in Fig. 3(d) 


\section{Conclusions}

In this paper we further applied the analytical method called the homotopy perturbation Shehu transform method (HPSTM) to some important fractional models arising in applied physical sciences. The analytical method proved to be highly efficient and does not require the unnecessary computations of the Adomian polynomials and the Lagrangian multiplier which is an advantage over the (ADM) and the (VIM). Using the analytical method, series solutions are easily computed and the results in closed form are successfully obtained. The obtained results are compared with the results of the existing techniques. The HPSTM proved to be a powerful mathematical tool for solving fractional models and can further be extended to more complex fractional models in applied science and engineering.

\section{Acknowledgement}

This research is partially supported by the National Natural Science Foundations of China (12071261, 12001539, 11831010, 11871068), the Science Challenge Project (TZ2018001), and the National Key Basic Research Program (2018YFA0703903). The first author also acknowledges the financial support of China Scholarship Council (CSC) (2017GXZ025381).

\section{References}

[1] Barman, H.K., Seadawy, A.R., Akbar, M.A., \& Baleanu, D. (2020). Competent closed form soliton solutions to the Riemann wave equation and the Novikov-Veselov equation. Results in Physics, 103131.

[2] Khalouta, A., \& Kadem, A. (2020). A new iterative natural transform method for solving nonlinear Caputo time-fractional partial differential equations. Jordan Journal of Mathematics and Statistics, 13(3), 459-476.

[3] Khalouta, A., \& Kadem, A. (2020). Numerical comparison of FNVIM and FNHPM for solving a certain type of nonlinear Caputo time fractional partial differential equations. Annales Mathematicae Silesianae, 34(2), 203-221.

[4] Khalouta, A., \& Kadem, A. (2020). Solutions of nonlinear time-fractional wave-like equations with variable coefficients in the form of Mittag-Leffler functions. Thai Journal of Mathematics, $18(1), 411-424$.

[5] Maitama, S., \& Zhao, W. (2020). Beyond Sumudu transform and natural transform: J-transform properties and applications. Journal of Applied Analysis and Computation, 10, 1223-1241.

[6] Sharma, D., Samra, G.S., \& Singh, P. (2020). Approximate solution for fractional attractor onedimensional Keller-Segel equations using homotopy perturbation sumudu transform method. Nonlinear Engineering, 9(1), 370-381.

[7] Ziane, D., Belgacem, R., \& Bokhari, A. (2019). A new modified Adomian decomposition method for nonlinear partial differential equations. Open Journal of Mathematical Analysis, 3(2), $81-90$. 
[8] Eltayeb, H., \& Bachar, I. (2020). A note on singular two-dimensional fractional coupled Burger's equation and triple Laplace Adomian decomposition method. Boundary Value Problems, 2020(2020), 1-17.

[9] Qin, Y., Khan, A., Ali, I., Al Qurashi, M., Khan, H., Shah, R., \& Baleanu, D. (2020). An efficient analytical approach for the solution of certain fractional-order dynamical systems. Energies, 13, $1-14$.

[10] Maitama, S., \& Zhao, W. (2021). New Laplace-type integral transform for solving steady heattransfer problem. Thermal Science, 25, 1-12.

[11] Maitama, S., \& Zhao, W. (2019). New integral transform: Shehu transform a generalization of Sumudu and Laplace transform for solving differential equations. International Journal of Analysis and Application, 17, 167-190.

[12] Belgacem, R, Baleanu, D, \& Bokhari, A. (2019). Shehu transform and applications to Caputo-fractional differential equations. International Journal of Analysis and Applications, 17, 917-927.

[13] Atangana, A., \& Baleanu, D. (2016). New fractional derivatives with non-local and non-singular kernel: Theory and application to heat transfer model. Thermal Science, 20(2), 763-769.

[14] Issa, A., \& Mensah, Y. (2020). Shehu transform: Extension to distributions and measures. Journal of Nonlinear Modeling and Analysis, (4), 495-503.

[15] Bekela, A.S., Belachew, M.T., \& Wole, G.A. (2020). A numerical method using Laplace-like transform and variational theory for solving time-fractional nonlinear partial differential equations with proportional delay. Advances in Difference Equations, 2020:586.

[16] Bokharia, A., Baleanu, D., \& Belgacema, R. (2020). Application of Shehu transform to Atangana-Baleanu derivatives. Journal of Mathematics and Computer Science, 20, 101-107.

[17] Aggarwal, S., Sharma, S.D., \& Gupta, A.R. (2019). Application of Shehu transform for handling growth and decay problems. Global Journal of Engineering Science and Research, 6, 190-198.

[18] Qureshi S., \& Kumar P. (2019). Using Shehu transform to solve fractional order Caputo type initial value problems. Journal of Applied Mathematics and Computational Mechanics, 28, 75-83.

[19] Ziane, D., \& Cherif, M.H. (2020). Combination of two powerful methods for solving nonlinear differential equations. Earthline Journal of Mathematical Sciences, 3, 121-138.

[20] Khan, H., Farooq, U., Rasool, S., Balaenu, D., \& Kumam, Poom. (2020). Analytical solution of (2+time fractional order) physical models, using modified decomposition method. Applied Sciences, 10, 122.

[21] Khalouta A., \& Kadem, A. (2020). A comparative study of Shehu variational iteration method and Shehu decomposition method for solving nonlinear Caputo time-fractional wave-like equations with variable coefficients. Application and Applied Mathematics, 15(1), 430-445.

[22] Akinyemi, L, \& Iyiola, O. (2020). Exact and approximate solutions of time-fractional models arising from physics via Shehu transform. Mathematical Method in Applied Sciences, DOI: 10.1002/mma.6484, 1-23.

[23] Khalouta, A., \& Kadem, A. (2019). A new method to solve fractional differential equations: Inverse fractional Shehu transform method. Applications and Applied Mathematics, 14, 926-941.

[24] Khalouta, A., \& Kadem, A. (2020). A new modification of the reduced differential transform method for nonlinear fractional partial differential equations. Journal of Applied Mathematics and Computational Mechanics, 19(3), 45-58.

[25] Maitama, S., \& Zhao, W. (2020). New homotopy analysis transform method for solving multidimensional fractional diffusion equations. Arab Journal of Basic and Applied Sciences, 27:1, 27-44. 